\title{
Blastomycosis hospitalizations in northwestern Ontario: 2006-2015
}

\author{
S Litvinjenko ${ }^{1 *}$, D Lunny²
}

\section{Abstract}

Background: Blastomycosis, caused by the organism Blastomyces dermatitidis, is an invasive fungal disease found in Central Canada and Central and Midwestern United States.

Objective: To describe trends in and epidemiology of hospitalized cases of blastomycosis reported among northwestern Ontario residents between 2006 and 2015.

Methods: Blastomycosis hospitalization data were extracted from the Discharge Abstract Database (DAD), accessed through IntelliHEALTH Ontario. The DAD includes administrative, clinical and demographic information on hospital discharges provided by the Canadian Institute for Health Information (CIHI). Blastomycosis records were identified using ICD-10 codes B40.0 to B40.9. Hospitalization rates were calculated for all of Ontario, and age-specific hospitalization rates were calculated for northwestern Ontario and analyzed by local health region, time and seasonality as well as presenting symptoms.

Results: There were 581 hospitalizations for blastomycosis reported in Ontario over this 10-year period. Of these, 245 (42\%) were from northwestern Ontario, although this region accounts for only $0.6 \%$ of the Ontario population. The average hospitalization rate for blastomycosis in northwestern Ontario was 35.0 per 100,000 per year. This rate varied from 1.7 in the Red Lake region to 57.9 in the Kenora region. The most common presentation was acute pulmonary symptoms. Men were 1.36 times more likely to be hospitalized for blastomycosis than were women (95\% confidence interval $[\mathrm{Cl}]: 1.06-1.75, P<0.05)$. Most hospitalizations were registered in the late fall months, suggesting blastomycosis exposure in the spring/summer season followed by a lengthy incubation period.

Conclusion: Areas of northwestern Ontario have high reported rates of blastomycosis. It is not known to what extent there are regional differences in other states and provinces. Interregional differences may warrant prioritizing strategies for blastomycosis prevention and control as well as additional research and surveillance.

\author{
Affiliations \\ ${ }^{1}$ Formerly at the Northwestern \\ Health Unit, Kenora, ON \\ ${ }^{2}$ Northwestern Health Unit, \\ Kenora, ON
}

*Correspondence: stefan. litvinjenko@mail.utoronto.ca

Suggested citation: Litvinjenko S, Lunny D. Blastomycosis hospitalizations in northwestern Ontario: 2006-2015. Can Commun Dis Rep. 2017;43(10):200-5. https://doi.org/10.14745/ccdr.v43i10a02

\section{Introduction}

Blastomycosis, caused by the organism Blastomyces dermatitidis, is an invasive fungal disease whose only known natural reservoir is in soil. Cases of blastomycosis have occurred mainly across the eastern areas of North America, in the provinces and states that border the Great Lakes (i.e., Ontario, Michigan, Wisconsin, Minnesota), but some have also been found in the midwestern United States and central Canada (1). Rarely have cases been reported outside North America.

Symptomatic disease is thought to occur in approximately $50 \%$ of cases (2), suggesting that healthy individuals are fairly resistant to the harmful progression of the invasive fungus. It is estimated that $70 \%$ of cases can be attributed to pulmonary blastomycosis, which usually presents as flu-like illness; the symptoms may be commonly misdiagnosed as other morbidities, for example, tuberculosis. Extrapulmonary disease most commonly manifests as cutaneous blastomycosis, but it can also occur in the skeletal, urogenital and central nervous systems. With appropriate antifungal and/or surgical treatment, the mortality rate of blastomycosis is between 5 to $10 \%$ (2).
The average incubation period of blastomycosis is generally accepted to be 30 to 45 days, although the range can be anywhere from 13 to 106 days, depending on inoculum size (3) and the form of the disease (4). Although blastomycosis infection occurs primarily through the inhalation of airborne spores, it can also occur, though rarely, through a puncture in the skin with infected material. Exposure to the fungal spores may increase during excavation and construction operations as well as during recreational activities that involve contact with soil near waterways as the moist and acidic soil environment is favourable to $B$. dermatitidis growth $(2,5)$. Aerosolization of spores occurs more readily from dry soil that is disturbed (2). Due to changing climatic conditions (i.e., rainfall, temperature and humidity) and the effects on soil composition (i.e., $\mathrm{pH}$ and organic content), isolating $B$. dermatitidis from the environment can be challenging $(2,3,6)$.

In Ontario, blastomycosis has not been on the list of reportable diseases since 1989 (due to limited transmissibility and few cases being reported), although the disease remains notifiable in the 
province of Manitoba (7). Blastomycosis is common in the central and midwestern United States (8) and remains a reportable illness in Arkansas, Louisiana, Michigan, Minnesota and Wisconsin (9). According to Health Canada, the annual incidence rate of blastomycosis across Ontario, Quebec, Manitoba and Saskatchewan is 0.62 cases per 100,000 population, with areas that have hospitalization rates of 0.3 to 0.6 cases per 100,000 population (10).

A 2005 study on blastomycosis in northwestern Ontario identified a high annual incidence rate of 17.0 cases per 100,000 population in 1989-2005 (Mann V, Limerick B, Wiebe L. Northwestern Health Unit blastomycosis cases, 1989 to 2005: preliminary analysis. 2005; Unpublished data). An earlier study over a shorter period (1997-1999) calculated an annual blastomycosis rate of 117.2 cases per 100,000 at the time of an outbreak in Kenora (11). Along with the rate of 404.9 per 100,000 people living on reserve, these rates are considered exceptionally high (11).

The objective of this study is to utilize hospitalization data from the past decade (2006-2015) to describe recent trends in the epidemiology of blastomycosis hospitalization in northwestern Ontario.

\section{Methods}

This analysis focuses on hospitalized cases of blastomycosis in northwestern Ontario. For the purposes of this report, the term "northwestern Ontario" refers to the Northwestern Health Unit (NWHU) catchment area. The NWHU catchment area is one of the 36 public health unit regions in Ontario. It serves a population of just under 82,000 across an area of 171,288 square kilometres (roughly one-fifth the size of the province). The area contains part of the Kenora District and all of the Rainy River District, and this analysis includes Kenora, Dryden, Red Lake, Sioux Lookout, Rainy River, Emo, Fort Frances and Atikokan. These regions include the named municipalities as well as any nearby smaller communities and First Nations reserves. There are 39 recognized First Nations in the NWHU catchment area; some are located around the main municipalities while others are more northern and less easily accessible.

\section{Inclusion criteria}

The study sample included hospitalization records for any form of blastomycosis diagnosed in Ontario residents between 2006 and 2015 using ICD-10 codes B40.0 to B40.9 as the primary diagnosis (12).

Location of hospitalizations were based on patients' residence rather than where they were hospitalized. For example, if a patient from northwestern Ontario was hospitalized in Toronto, the hospitalization would be classified as northwestern Ontario. Hospitalizations that occurred outside of Ontario, however, were not captured in the data and could not be assessed.

\section{Data sources}

Blastomycosis hospitalization data were extracted on January 20, 2017 from the Discharge Abstract Database (DAD). The DAD was accessed through IntelliHEALTH Ontario, a knowledge repository operated by the Ministry of Health and Long-Term Care. The DAD includes administrative, clinical and demographic information on hospital discharges provided by the Canadian
Institute for Health Information (CIHI). IntelliHEALTH Ontario is operated by the Ontario Ministry of Health and Long-Term Care.

Population estimates for the NWHU catchment area came from Statistics Canada and were accessed through IntelliHEALTH Ontario.

\section{Hospitalization counts and rates}

Counts of blastomycosis hospitalizations for each of Ontario's 36 public health units were tabulated. From these, hospitalization rates were derived for residents of northwestern Ontario as well as from smaller geographical regions within this area.

Crude and age-specific hospitalization rates were calculated by dividing the number of hospitalizations occurring over the time period by the total person-years at risk, and multiplying the result by 100,000 person-years. A rate displayed in 100,000 person-years indicates the number of hospitalizations that occur in a population of 100,000 people over the course of one year.

Age-standardized hospitalization rates were calculated using the 2011 Canadian Census population as the reference population. All analyses were conducted using Microsoft Excel, Open Epi and Epilnfo7.

\section{Northwestern Ontario rates}

Age-standardized hospitalization rates for the population within the NWHU catchment and the subregions within it as well as the corresponding $95 \%$ confidence intervals $(\mathrm{Cls})$ were calculated using the Poisson approximation of the normal distribution. Rate ratios were calculated and used to identify any statistical differences in hospitalization rates between the subregions. Results were considered statistically significant if the $95 \% \mathrm{Cl}$ around the rate ratio did not contain one.

\section{Rates by age and sex}

Age- and sex-specific hospitalization rates and 95\% Cls were calculated using the Mid-P Exact method. Ten-year age groups were used for the analysis $(0-9,10-19$, etc.). Tests for statistical differences in rates between age and sex strata were carried out by calculating the rate ratios. Results were considered statistically significant if the $95 \% \mathrm{Cl}$ around the rate ratio did not contain one.

\section{Counts by diagnosis code}

Counts of hospitalizations categorized by type of blastomycosis were tabulated for northwestern Ontario. Counts for each type of blastomycosis were based on ICD-10 diagnosis codes, which ranged from B40.0 to B40.9 (12).

\section{Counts by year and month}

Counts of hospitalizations were examined by year and month of occurrence. Cumulative month totals over the 10-year study period were calculated to determine when in the year hospitalizations were the most common.

\section{Results}

\section{Blastomycosis hospitalizations in Ontario}

Between 2006 and 2015, a total of 581 blastomycosis hospitalizations were recorded by 29 of Ontario's 36 public 
health units (the remaining seven health units registered no cases). Notably, 245 blastomycosis hospitalizations (42\%) were attributed to residents of northwestern Ontario alone (i.e., in the NWHU) (Figure 1).

Figure 1: Number of blastomycosis hospitalizations by public health unit, Ontario, 2006-2015 $(n=581)$

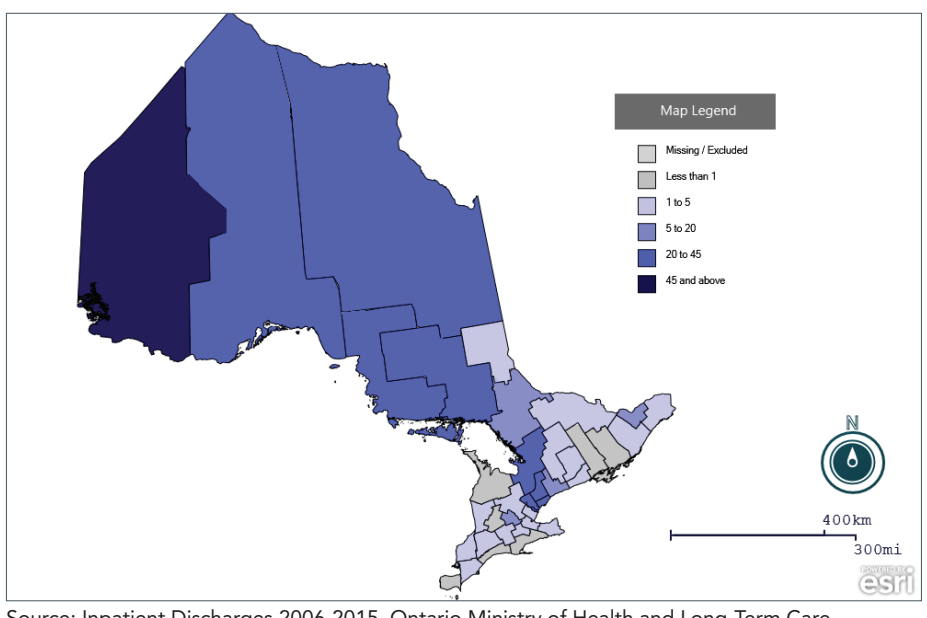

Source: Inpatient Discharges 2006-2015. Ontario Ministry of Health and Long-Term Care. IntelliHEALTH Ontario. Date Extracted: January 10, 2017

\section{Hospitalizations in northwestern Ontario by region}

In northwestern Ontario, the hospitalization rate for blastomycosis was 35.0 per 100,000 per year between 2006 and 2015. Kenora had the highest rate of hospitalizations, at 57.9 per 100,000 per year, and a statistically significant rate ratio of 3.13 (95\% Cl: $2.42-4.09 ; P<0.01$ ) compared with the rest of northwestern Ontario. Due to small counts, hospitalizations in the Atikokan, Emo and Red Lake subregions have been suppressed and are not reported on. Other regions in the area had hospitalizations rates that ranged from 16 to 32 per 100,000 per year (Figure 2).

Figure 2: Blastomycosis hospitalizations* by northwestern Ontario region ${ }^{\dagger}, 2006-2015$

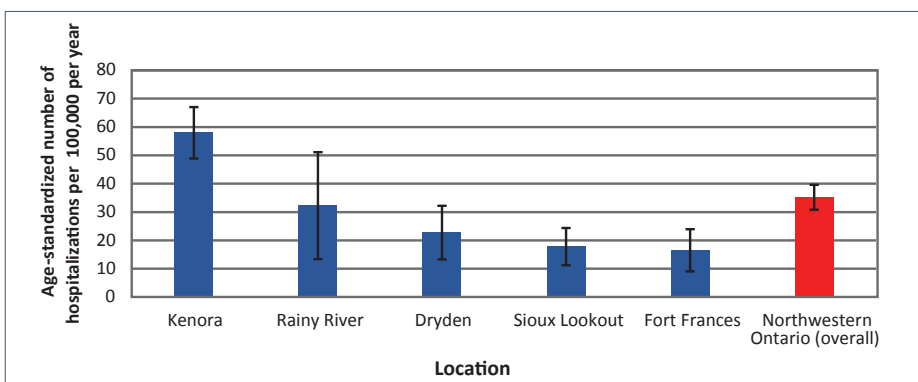

* Rates per 100,000 per year age standardized, with $95 \%$ confidence intervals

+ Regions include the named municipality in addition to smaller nearby communities and First Nation reserves

Note: Results from Red Lake, Emo and Atikokan were omitted due to small counts Source: Inpatient Discharges 2006-2015. Ontario Ministry of Health and Long-Term Care. IntelliHEALTH Ontario. Date Extracted: January 10, 2017

\section{Hospitalizations in northwestern Ontario by age and sex}

Males, who represented $50.3 \%$ of the population in the NWHU catchment area between 2006 and 2015, accounted for 58\%
( $n=142)$ of all blastomycosis hospitalizations (compared with $n=103$ for females). The rate of blastomycosis hospitalizations for males of all ages was 34.4 per 100,000 per year, compared with 25.3 per 100,000 per year for females. This equals a statistically significant rate ratio of $1.36(95 \% \mathrm{Cl}: 1.06-1.76 ; P<0.05)$.

Blastomycosis hospitalizations were lowest among children aged less than 10 years and adults aged 60 years and older. Rates were generally highest among adults in their twenties to their fifties (Figure 3). People in the 30- to 39-year age category had a statistically significant rate ratio of 2.04 (95\% Cl: $1.49-2.76$; $P<0.01)$ compared with the other age categories. However, examination of the data showed that overrepresentation of males inflated this overall estimate; the rate ratio among men aged 30-39 years was 2.51 (95\% Cl: 1.70-3.66; $P<0.01)$ compared with females in this age group, among whom rates were insignificant.

Figure 3: Blastomycosis hospitalization rates ${ }^{\star}$ by age and sex, northwestern Ontario, 2006-2015

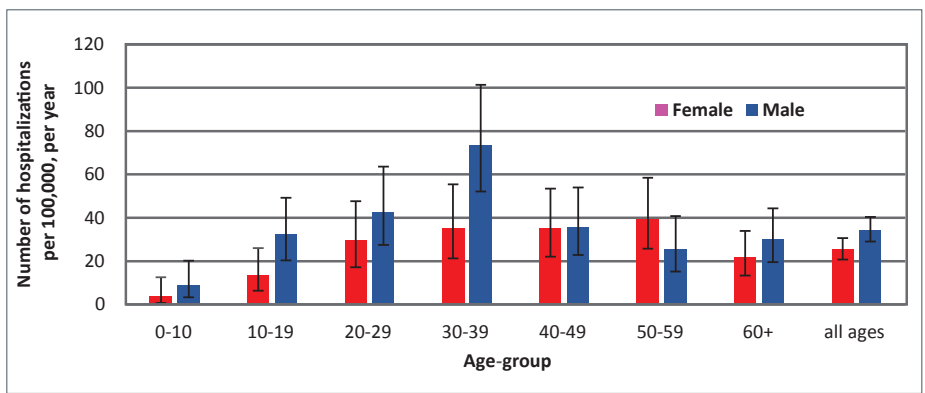

*Rates per 100,000 per year with $95 \%$ confidence intervals

Source: Inpatient Discharges 2006-2015. Ministry of Health and Long-Term Care. IntelliHEALTH Ontario. Date Extracted: January 10, 2017

\section{Hospitalizations in northwestern Ontario by ICD-10 diagnosis code}

The majority of blastomycosis hospitalizations between 2006 and 2015 (75\% of total cases), were due to pulmonary illness (Figure 4$)$. While the nature of some cases ( 10\%) is unspecified, it can be assumed that most would also have been attributed to acute pulmonary infection indicative of temporary infection. This differs from the more infectious relapsing chronic condition, which accounts for $2.5 \%$ of known hospitalizations. Other manifestations of blastomycosis disease, including cutaneous and disseminated varieties, accounted for less than $5 \%$ of known hospitalizations.

Figure 4: Blastomycosis hospitalizations by ICD-10 diagnosis code, northwestern Ontario, 2006-2015

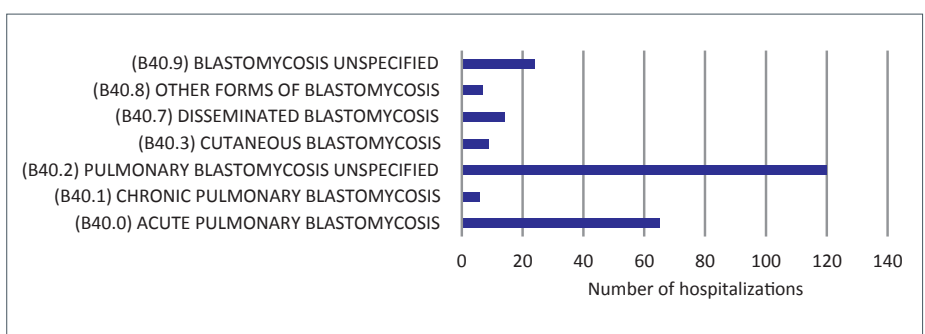

Source: Inpatient Discharges 2006-2015. Ontario Ministry of Health and Long-Term Care. IntelliHEALTH Ontario. Date Extracted: January 10, 2017 


\section{Hospitalizations in northwestern Ontario by year and month}

There was a slight increase in the number of blastomycosis hospitalizations in northwestern Ontario between 2006 and 2015 (Figure 5). Aside from the general increasing trend in hospitalization counts, 2009 and 2013 were noted as peak years, both with 41 hospitalizations.

Figure 5: Blastomycosis hospitalizations in northwestern Ontario by year, 2006-2015

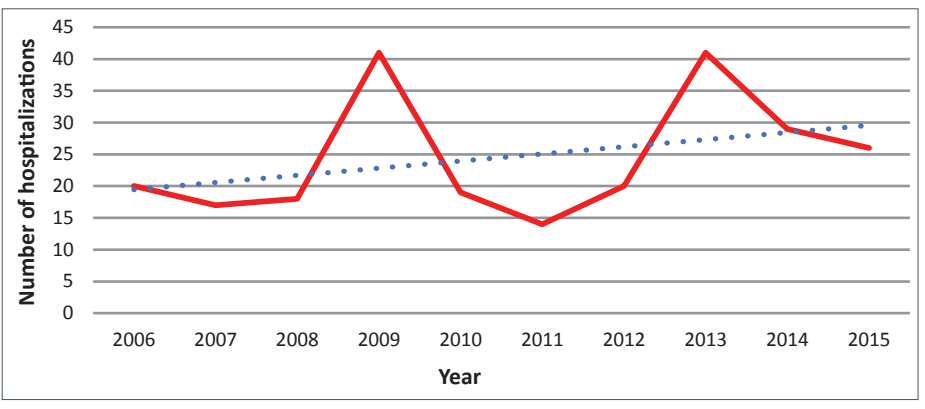

Source: Inpatient Discharges 2006-2015. Ontario Ministry of Health and Long-Term Care. IntelliHEALTH Ontario. Date Extracted: January 10, 2017

Between 2006 and 2015, there were more hospitalizations in the late fall to early winter months, on average, than during any other time of the year (Figure 6).

Figure 6: Blastomycosis hospitalizations in northwestern Ontario by month, 2006-2015 ( $n=245)$

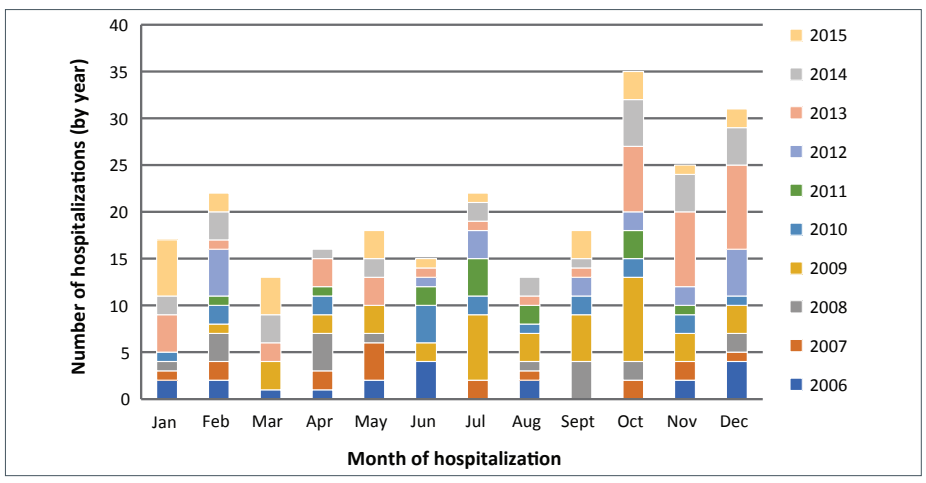

Source: Inpatient Discharges 2006-2015. Ontario Ministry of Health and Long-Term Care. IntelliHEALTH Ontario. Date Extracted: January 10, 2017

\section{Discussion}

Between 2006 and 2015, almost 600 hospitalizations for blastomycosis were reported in Ontario. Based on 2015 population estimates, northwestern Ontario accounts for only $0.6 \%$ of the provincial population and yet over $40 \%$ of total hospitalizations over the period of the study. These rates are likely to be an underestimate of the true incidence of blastomycosis, as the literature suggests that only about $50 \%$ of people with blastomycosis present clinical symptoms for hospitalization (13). There appeared to be a lot of regional variation even within northwestern Ontario, with certain health regions reporting zero cases over this 10 -year period, while the Kenora region consistently demonstrated one of the highest hospitalization rates for blastomycosis in the province, driving regional/provincial estimates of morbidity. Coupled with previously identified estimates from the literature, high rates in the Kenora region may be due to geological differences compared to eastern and southern parts of northwestern Ontario, which may result in better soil conditions for blastomycosis, although soil types were not tested as part of this study. It is not known whether such regional variations are also characteristic of other Canadian provinces or the United States (US).

Furthermore, our finding of 35.0 per 100,000 per year hospitalization rate for northwestern Ontario remain well above the highest average annual rate reported in any US state but overlaps with some regional data in the US. In Wisconsin, for example, although there was an age-adjusted rate of 2.9 hospitalizations per 100,000 reported for the state (16), there were reports of $10-40$ cases per 100,000 in certain Wisconsin counties (17). It should be noted that average annual increases in blastomycosis rates in the northwestern Ontario might be indicative of genuine increases of incidence of the disease, or reflect improved clinical awareness and testing from physicians (12).

That the majority of blastomycosis cases in Ontario in 2006-2015 were male (58\%) is consistent with data from other studies. This finding can be attributed to men dominating the types of labour (such as excavation or construction) common in these areas. More men than women may also prefer recreational activities near waterways that favour growth of $B$. dermatitidis (5). The exception was in the 50 - to 59-year age group, where $59 \%$ of cases were female and $41 \%$ male.

Most blastomycosis hospitalizations were in the 30- to 39year age group. This is in contrast to Manitoba where 50- to 69-year-olds shared the highest incidence rate (2). Similarly, an older report from northwestern Ontario found the highest hospitalization rates among people in the 40- to 59-year age group (Mann $V$ et al unpublished data). Irrespective of age and sex, seasonality trends are consistent with exposure in the spring/summer season and an incubation period that would lead to diagnosis in the late fall.

\section{Strengths and limitations}

A strength of this study was the quality of the $\mathrm{ClHI}$ data in its capacity to detect regional differences in disease and to provide a comprehensive hospitalization-based sample population (a large proportion of blastomycosis literature is based on outbreak-specific reporting). In terms of validity, Public Health Ontario supplied the NWHU with count data of laboratory-confirmed positive cultures of $B$. dermatitidis for the years 2010-2015, which in theory would be a more representative measure of blastomycosis incidence in the region. However, as the hospitalization data yielded numbers comparable to the laboratory-confirmed count data, the hospitalization data were considered an adequate proxy measure for blastomycosis-associated hospitalization in northwestern Ontario.

A limitation of the study is the use of hospitalization records to assess blastomycosis, which can only serve as a proxy for true incidence, given that not everyone can be expected to seek and/or have access to care or be hospitalized if they do seek medical care. In addition, some cases, particularly severe cases, are referred to Winnipeg, Manitoba; as a result they would not be captured as an Ontario case, and may potentially confound incidence rates in Manitoba between locally attributed exposures, and out of province visitations (14). Moreover, while other studies found significantly higher rates of blastomycosis in Indigenous populations $(11,14,15)$, information 
on the Indigenous status of the cases was not available in our study population. Future studies assessing individualized case-record data, the monitoring of relevant risk factors (e.g., socio-demographic factors), isolating probable sources of infection, and determining overall rates of incidence would help address some of the limitations of the current study and increase our understanding of this disease.

\section{Conclusion}

Areas of northwestern Ontario have high reported rates of blastomycosis. It is not known to what extent there are regional differences in other states and provinces. Interregional differences may warrant prioritizing strategies for blastomycosis surveillance, prevention and control, as well as additional research.

\section{Authors' statement}

SL - Conceptualization, Methodology, Formal Analysis, Investigation, Visualization, Writing - original draft, Writing review and editing, Project administration

DL - Conceptualization, Methodology, Validation, Resources, Data Collection, Writing - review and editing, Supervision, Project administration

\section{Conflict of interest}

None.

\section{Acknowledgements}

We would like to thank Dr. Kit Young-Hoon, Medical Officer of Health for the Northwestern Health Unit, for her supervision, expertise and validation of this report. An additional thank you to all of those in the field who helped collect these data and who provide care to those with infectious diseases.

\section{Funding}

This research was supported by the Northwestern Health Unit.

\section{References}

1. Centers for Disease Control \& Prevention. Blastomycosis. Atlanta (GA): CDC. https://www.cdc.gov/fungal/diseases/ blastomycosis/ [Accessed 2017 Feb 8].

2. Manitoba Health Communicable Disease Control Unit. Communicable Disease Management Protocol: Blastomycosis. 2015. https://www.gov.mb.ca/health/ publichealth/cdc/protocol/blastomycosis.pdf

3. Klein BS, Vergeront JM, Weeks RJ, Kumar UN, Mathai G, Varkey B, Kaufman L, Bradsher RW, Stoebig JF, Davis JP. Isolation of Blastomyces dermatitidis in soil associated with a large outbreak of blastomycosis in Wisconsin. N Eng J Med. 1986;314:529-34. DOI (http://dx.doi.org/10.1056/
NEJM198602273140901). PubMed (https://www.ncbi.nlm. nih.gov/entrez/query.fcgi?cmd=Retrieve $\& d b=$ PubMed $\&$ li st_uids=3945290\&dopt=Abstract)

4. Gray NA, Baddour LM. Cutaneous inoculation blastomycosis. Clin Infect Dis. 2002;34(10):E44-9. DOI (http://dx.doi. org/10.1086/339957). PubMed (https://www.ncbi.nlm.nih. gov/pubmed/11981746?dopt=Abstract).

5. Klein BS, Vergeront JM, DiSalvo AF, Kaufman L, Davis JP. Two outbreaks of blastomycosis along rivers in Wisconsin: isolation of Blastomyces dermatitidis from riverbank soil and evidence of its transmission along waterways Am Rev Respir Dis. 1987;136(6):1333-8. DOI (http://dx.doi.org/10.1164/ ajrccm/136.6.1333). PubMed (https://www.ncbi.nlm.nih. gov/entrez/query.fcgi?cmd=Retrieve $\& \mathrm{db}=$ PubMed\&lis t_uids=3688635\&dopt=Abstract).

6. Morris SK, Brophy J, Richardson SE, Summerbell R, Parkin PC, Jamieson F, Limerick B, Wiebe L, Ford-Jones EL. Blastomycosis in Ontario, 1994-2003. Emerg Infect Dis.,2006;12(2):274-9. DOI (http://dx.doi.org/10.3201/ eid1202.050849). PubMed (https://www.ncbi.nlm.nih. gov/entrez/query.fcgi?cmd=Retrieve \&db=PubMed\&lis t_uids=16494754\&dopt=Abstract)

7. Population and Public Health Branch Summary of reportable diseases 1990. Toronto: Communicable Disease Control, Ontario Ministry of Health; 1991.

8. Seitz AE, Adjemian J, Steiner CA, Prevots DR Spatial epidemiology of blastomycosis hospitalizations: detecting clusters and identifying environmental risk factors. Med Mycol. 2015 Jun;53(5):447-54. DOI (http://dx.doi. org/10.1093/mmy/myv014). PubMed (https://www.ncbi.nlm. nih.gov/entrez/query.fcgi?cmd=Retrieve \&db=PubMed\&li st_uids=25908653\&dopt=Abstract).

9. Centers for Disease Control \& Prevention. Reportable fungal diseases by state. Atlanta (GA): CDC. https://www.cdc.gov/ fungal/fungal-disease-reporting-table.html [Accessed 2017 Feb 8].

10. Government of Canada. Surveillance of blastomycosis. Ottawa (ON): Health Canada. https://www.canada.ca/en/ public-health/services/diseases/blastomycosis/surveillanceblastomycosis.html [Accessed 2017 Feb 8].

11. Dwight PJ, Naus M, Sarsfield P, Limerick B. An outbreak of human blastomycosis: the epidemiology of blastomycosis in the Kenora Catchment Region of Ontario, Canada. Can Commun Dis Rep. 2000;26(10):82-91. PubMed (https://www. ncbi.nlm.nih.gov/entrez/query.fcgi?cmd=Retrieve\&db=PubM ed\&list_uids=10893821\&dopt=Abstract).

12. World Health Organization. International statistical classification of diseases and health related problems, 10th revision. Geneva: World Health Organization. 2016.

13. Chapman SW, Dismukes WE, Proia LA, Bradsher RW, Pappas PG, Threlkeld MG, Kauffman CA; Infectious Diseases Society of America. Clinical practice guidelines for the management of blastomycosis: 2008 update by the Infectious Diseases Society of America. Clin Infect Dis. 2008;46(12):1801-12. DOI (http://dx.doi.org/10.1086/588300). PubMed (https:// www.ncbi.nlm.nih.gov/entrez/query.fcgi?cmd=Retrieve\&db= PubMed\&list_uids=18462107\&dopt=Abstract). 
14. Crampton TL, Light RB, Berg GM, Meyers MP, Schroeder GC, Hershfield ES, Embil JM. Epidemiology and clinical spectrum of blastomycosis diagnosed at Manitoba hospitals. Clin Infect Dis. 2002;34(10):1310-6. DOI (http://dx.doi. org/10.1086/340049). PubMed (https://www.ncbi.nlm.nih. gov/entrez/query.fcgi?cmd=Retrieve\&db=PubMed\&lis t_uids=11981725\&dopt=Abstract).

15. Dalcin D, Ahmed S. Blastomycosis in northwestern Ontario, 2004 to 2014. Can J Infect Dis Med Microbiol. 2015;26(5):259-62. DOl (http://dx.doi. org/10.1155/2015/468453). PubMed (https://www.ncbi.nlm.
nih.gov/entrez/query.fcgi?cmd=Retrieve\&db=PubMed\&li st_uids=26600814\&dopt=Abstract).

16. Seitz A, Younes N, Steiner C, Prevots D. Incidence and trends of blastomycosis-associated hospitalizations in the United States. PLoS One. 2014;9(8):e105466. DOI (http://dx.doi. org/10.1371/journal.pone.0105466). PubMed (https://www. ncbi.nlm.nih.gov/entrez/query.fcgi?cmd=Retrieve\&db=PubM ed\&list_uids=25126839\&dopt=Abstract).

17. Benedict K, Roy M, Chiller T, Davis J. Epidemiologic and Ecologic features of blastomycosis: a review. Curr Fungal Infect Rep. 2012;6(4):327-35. DOI (http://dx.doi.org/10.1007/ s12281-012-0110-1).
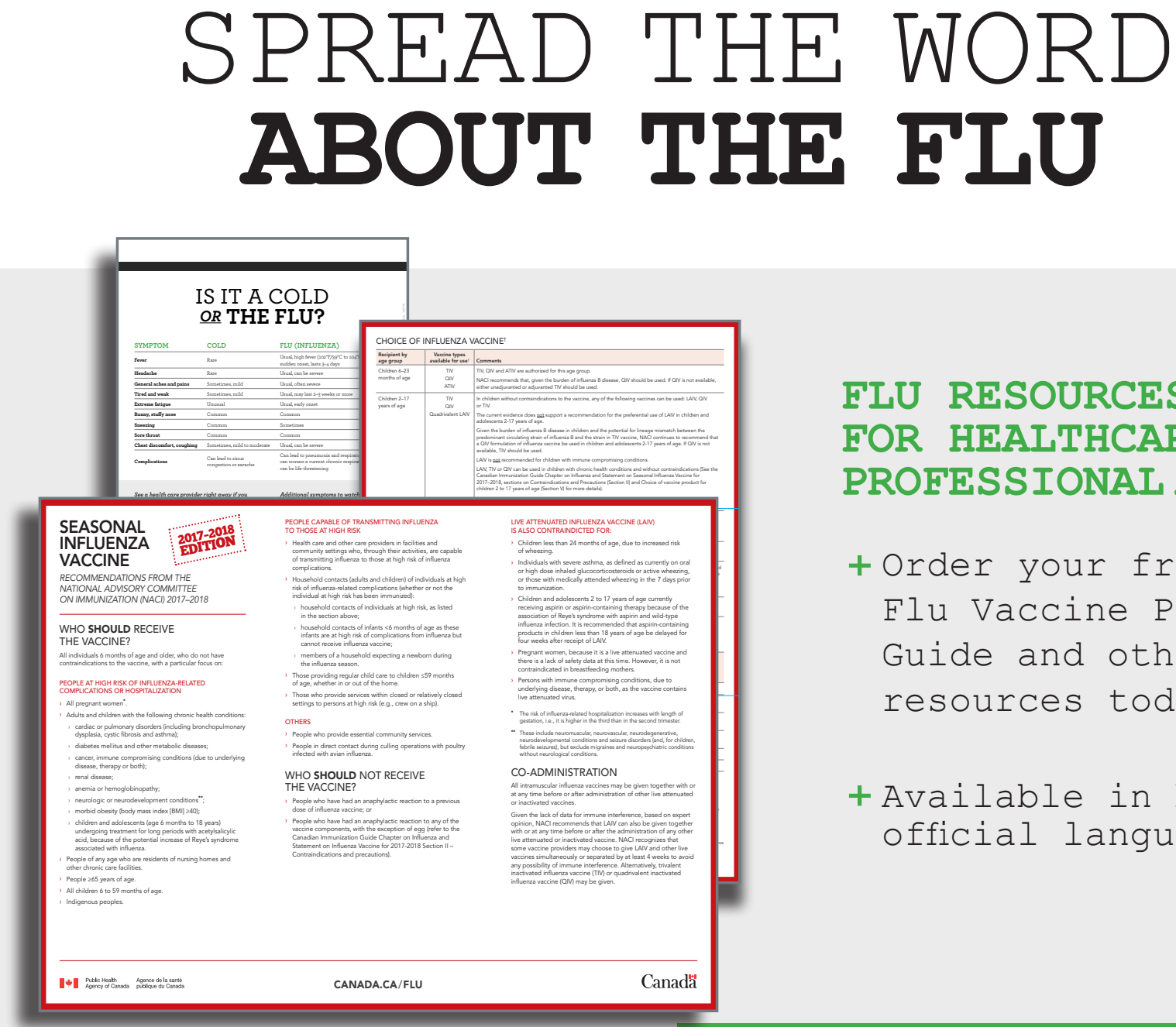

\section{FLU RESOURCES FOR HEALTHCARE PROFESSIONAL .}

+ Order your free Flu Vaccine Pocket Guide and other flu resources today

+ Available in both official languages

\section{PLACE YOUR ORDER TODAY. VISIT CANADA.CA/FLU}

